\title{
PassItOn: An Opportunistic Messaging Prototype on Mobile Devices
}

\author{
Bo Xing ${ }^{1}$, Karim Seada ${ }^{2}$, Péter Boda ${ }^{2}$, and Nalini Venkatasubramanian ${ }^{1}$ \\ ${ }^{1}$ School of Information and Computer Sciences, University of California, Irvine \\ ${ }^{2}$ Nokia Research Center, Palo Alto
}

\section{INTRODUCTION}

With the increasing popularity of mobile handheld devices and the growing capability of these devices, it is becoming possible that information sharing/dissemination is carried out through human networks, as a complement to the traditional computer networks. In such human networks, people come across one another, while their mobile devices exchange and store information in a spontaneous and transparent way. Such an encounter could be established through direct device-todevice connectivity when two devices come into each other's communication range, or be enabled by, e.g., a Wi-Fi access point, when the devices both enter its coverage.

A new form of dissemination, which we call opportunistic messaging, is such an application that is based on human encounters and mobilities. When human encounters are exploited for communications, the reliance on network infrastructure access is eliminated; communications can be performed even where infrastructure is absent or infrastructure access is intermittent. By leveraging human mobilities, data delivery does not require an end-to-end path from the source to a recipient; instead, people carrying mobile devices serve as relays - they cache others' data and forward/deliver the data when appropriate. Thus, the propagation of information is tied to people's physical proximity when they move around, and incorporates the social aspects of communications as people tend to spend more time co-locating with their social relations.

Opportunistic messaging is applicable anywhere, and is especially appealing where network infrastructure access is limited or intermittent (e.g., on cruise ships, in national parks, after disasters). Another intriguing characteristic of it is its ease of deployment - no central server is needed, but only a single piece of software on users' mobile devices. However, as human encounters and mobilities are unpredictable, when used for social applications, opportunistic messaging is most suited for disseminating user-generated information that is non-formal, less important, and thus not time-critical.

In recent years, a considerable amount of efforts have been invested in the research on opportunistic networking and delay-tolerant networking (which encompasses opportunistic networking but is a broader concept). A large portion of prior work has focused on routing issues, e.g., through whom as intermediate carriers to deliver a message to the destinations [1] [2] [3] [4]. The routing issues have been further explored in various contexts, such as in vehicular networks [5] [6] [7] and in social networking applications [8] [9] [10] [11]. However, serious real-world application development, deployment and evaluation of the opportunistic networking concepts still fall behind [12], in which many challenging issues remain to be addressed (to name a few, location-awareness, user incentives and preferences, power preservation, encounter controls, etc.).

In this work, we design and prototype PassItOn, a fully distributed opportunistic messaging system. Our goal is to build up a proof-of-concept platform on real mobile devices, and thus show the feasibility and potentials of utilizing human movements for dissemination applications. Meanwhile, we seek to shed lights on the design, implementation and deployment issues in building such systems, and thus stimulate new ideas and perspectives on addressing these issues. Moreover, we aim to offer a real testbed on which new mechanisms, protocols and use cases can be tested and evaluated.

\section{Passiton: The Prototype}

PassItOn is implemented on the Symbian S60 platform 3rd version [13] using the Python for S60 programming language and APIs [14]. Some Python extension modules written in C++ are created to access certain advanced operations in Symbian. The mobile devices we use are the Nokia N95 8GB smart phones [15]. For establishing encounters between the devices, multiple connectivities (Bluetooth, Wi-Fi infrastructure mode, Wi-Fi ad-hoc mode) are used. To achieve the best dissemination performance and to preserve energy, one of them is selected for use at a time depending on their performance tradeoffs.

From application point of view, the current version of PassItOn offers the following functionalities. (i) Interest matching. Users are able to see who else have similar interests as they do (for example in an office scenario, who have similar lunch preferences). (ii) Multiple forms of messaging. Users are able to send messages through unicast (one to one), multicast (one to many) or broadcast (one to all). (iii) Questions and answers. Users are able to raise questions, which will be automatically directed to those who likely will be able to answer the questions, and get them answered.

In order to accommodate the various communication forms and take into account the social, temporal and spatial aspects of communications, PassItOn is designed on basis of a generic messaging framework we come up with. A source device is able to specify the destination, the time relevance and the location relevance of a dissemination. The destination can be of the following types: (i) specific persons, (ii) all people with 
particular interests, (iii) given number of people with particular interests, (iv) all people, and (v) given number of people. The time relevance of a dissemination indicates the lifetime of the content, which will be removed from the system after its lifetime expires. The location relevance of a dissemination dictates the location where potential recipients should receive the content. A dissemination could be location-independent, or of the following types: (i) the static location where the source initiates the dissemination, (ii) the dynamic location of the source while she moves around, (iii) the expanding location which covers the source's moving path.

Under our generic messaging framework, we apply the publish/subscribe paradigm to PassItOn, in which any user can be a content publisher, a recipient, and a carrier, while any attribute of a user can be a subscription (interests, addresses, or even presences). This makes sense, because in many realworld sharing/dissemination scenarios, it is the recipients of the information (rather than the source) who determines whether and how to seize the information. When the source initiates a dissemination, she might not have knowledge of who will be exploiting the information.

The Routing/forwarding mechanism employed in PassItOn is adapted to our generic messaging framework, and moreover takes location relevance into consideration. During encounters, forwarding decisions for each message are made on basis of two values: (a) social utility and (b) location stickiness. Social utility captures how well a device is socially tied with the addressees of the message (similar to SocialCast [11] and Delegation Forwarding [16]). Location stickiness represents how likely a device will stick to the relevant location of the message. A device with higher location stickiness and social utility is considered as a better carrier of the message.

In PassIOn, a user has flexibility in specifying her preferences in several aspects: (i) how many messages she is willing to carry for others, (ii) for whom she is willing/unwilling to carry messages, (iii) under what battery level she is unwilling to carry messages for others, (iv) how she would like received messages to be presented, etc.. Based on that, an incentive mechanism is implemented, which encourages users to carry messages for others through granting virtual credit points. It determines whether a user can publish or retrieve messages based on the number of credit points she has.

\section{THE DEMONSTRATION}

In this demonstration, we run PassItOn on a few Nokia N95 $8 \mathrm{~GB}$ smart phones, and show how it achieves multiple forms of dissemination based on human encounters and mobilities, with social, temporal and spatial aspects being taken into account. The phones all have the PassItOn mobile client software preinstalled. Their Bluetooth interfaces are activated. When using Wi-Fi for establishing encounters, they periodically transmit HELLO beacons to advertise their presence.

We show how users can subscribe their interests, view others' interests, specify their preferences, and publish contents for various types of recipients. Moreover, we specifically demonstrate the following scenarios. (i) Sending messages to people out
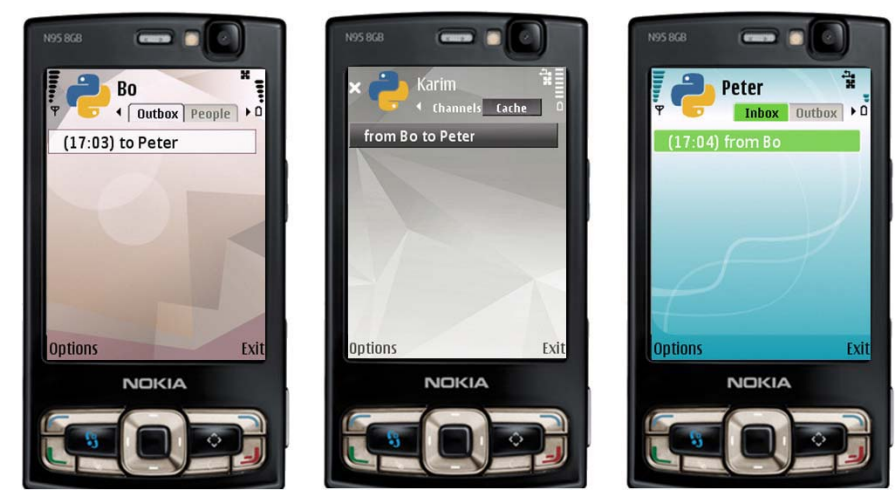

Fig. 1. Snapshots of the user interfaces in a demo scenario (Bo initiates a message addressed to Peter, who is out of direct contact of Bo. Karim, who meets Peter more often, carries the message from Bo, and delivers it to Peter when he encounters Peter again.)

of reach. In this scenario, we show how human encounters and mobilities aid data forwarding/delivery from information sources to destinations between which no end-to-end path exists at any point of time. We also show the roles that social ties derived from people's encounter histories play in facilitating efficient data forwarding. (ii) Leaving messages to a location. In this scenario, we show how a message can be attached to the sender's location, and the addressees can get the message when they enter the location even without the sender being present. We also show the impact of devices' location stickiness on data forwarding decision making. Some snapshots of the demonstration are shown in Fig. 1.

Our demonstration has minimum requirements in terms of equipments, network access, space and setup time. Only the phones are needed, even without SIM cards inserted.

\section{REFERENCES}

[1] M. Musolesi, S. Hailes, and C. Mascolo, "Adaptive routing for intermittently connected mobile ad hoc networks," in WoWMoM, 2005.

[2] J. Leguay, T. Friedman, and V. Conan, "Evaluating mobility pattern space routing for dtns," in Infocom, 2006.

[3] C. Boldrini, M. Conti, I. Iacopini, and A. Passarella, "Hibop: a history based routing protocol for opportunistic networks," in WoWMoM, 2007.

[4] A. Balasubramanian, B. N. Levine, and A. Venkataramani, "Dtn routing as a resource allocation problem," in SIGCOMM, 2007.

[5] J. LeBrun, C.-N. Chuah, D. Ghosal, and M. Zhang, "Knowledge-based opportunistic forwarding in vehicular wireless ad hoc networks," in VTC, 2005.

[6] I. Leontiadis and C. Mascolo, "Geopps: Geographical opportunistic routing for vehicular networks," in AOC, 2007.

[7] — , "Opportunistic spatio-temporal dissemination system for vehicular networks," in MobiOpp, 2007.

[8] E. Daly and M. Haahr, "Social network analysis for routing in disconnected delay-tolerant manets," in MobiHoc, 2007.

[9] A. Chaintreau, P. Hui, J. Crowcroft, C. Diot, R. Gass, and J. Scott, "Impact of human mobility on the design of opportunistic forwarding algorithms," in IEEE Transactions on Mobile Computing, 2007.

[10] P. Hui, J. Crowcroft, and E. Yoneki, "Bubble rap: Social-based forwarding in delay tolerant networks," in MobiHoc, 2008.

[11] P. Costa, C. Mascolo, M. Musolesi, and G. P. Picco, "Socially-aware routing for publish-subscribe in delay-tolerant mobile ad hoc networks," in IEEE Journal On Selected Areas In Communications (JSAC), 2008.

[12] H. Yoon, J. Kim, F. Tan, and R. Hsieh, "On-demand video streaming in mobile opportunistic networks," in PerCom, 2008.

[13] "S60", http: / /www. s60.com.

[14] "Pys60," wiki.opensource.nokia.com/projects/Pys60.

[15] "Nokia n95 8gb," https: //www.nokiausa.com/A4513447.

[16] V. Erramilli, M. Crovella, A. Chaintreau, and C. Diot, "Delegation forwarding," in MobiHoc, 2008. 